Revue européenne des sciences sociales

European Journal of Social Sciences

XLV-138 | 2007

Évaluation en sciences sociales. Concepts, mesures et comparaisons

\title{
Classifications, typologies et rapport aux valeurs
}

Jacques Coenen-Huther

\section{(2) OpenEdition}

Journals

Édition électronique

URL : http://journals.openedition.org/ress/191

DOI : $10.4000 /$ ress. 191

ISSN : 1663-4446

Éditeur

Librairie Droz

Édition imprimée

Date de publication : 2 juillet 2007

Pagination : $27-40$

ISBN : 2-600-01170-9

ISSN : 0048-8046

Référence électronique

Jacques Coenen-Huther, "Classifications, typologies et rapport aux valeurs », Revue européenne des sciences sociales [En ligne], XLV-138 | 2007, mis en ligne le 01 juillet 2010, consulté le 10 décembre 2020. URL : http://journals.openedition.org/ress/191 ; DOI : https://doi.org/10.4000/ress.191 


\section{CLASSIFICATIONS, TYPOLOGIES ET RAPPORT AUX VALEURS}

Tout projet scientifique se fonde sur des opérations de classification plus ou moins élaborées. Celles-ci plongent leurs racines dans les procédés empiriques de la connaissance ordinaire mais prennent une place décisive dans les diverses procédures scientifiques dont elles constituent la condition sine qua non. Appliquer son esprit à l'examen d'une question quelconque, dans un but de connaissance ou d'action, c'est se mettre plus ou moins consciemment à classer et à comparer, le classement étant le préalable de la comparaison ${ }^{1}$. En se plaçant à ce niveau d'abstraction et de généralité, on est fondé à considérer comme semblables les procédés de raisonnement des sciences de la nature et ceux des sciences humaines. Les exposés de méthodologie sociologique renforcent d'ailleurs souvent le sentiment d'unité de la démarche scientifique. Demazière et Dubar, traitant de l'élaboration de typologies, y voient «une des opérations les plus courantes et les plus pratiquées dans les sciences sociales comme dans les sciences expérimentales». Il s'agit selon eux, dans les premières comme dans les secondes, de «mettre de l'ordre dans les matériaux recueillis, les classer selon des critères pertinents, trouver les variables cachées qui expliquent les variations des diverses dimensions observables (1997, p. 274).

\section{LA RÉDUCTION DE COMPLEXITÉ}

Dans le domaine des sciences humaines comme dans celui des sciences de la nature, ce désir de «mettre de l'ordre» ou de «voir clair» se fonde sur « une tradition d'intelligibilité par la classification» remontant à Aristote (Schnapper, 2005, p. 15) mais qui s'est transformée en un véritable «mode d'explication scientifique» à l'époque des Lumières (Valade, 2003, pp. 123-124). Cette conception de l'activité scientifique entraîne une volonté de réduction de complexité qui est apparente dans les classifications de Buffon ou de Linné mais qu'on retrouve chez Durkheim. Dans le chapitre IV des Règles de la méthode sociologique, celui-ci, s'inspirant fortement des procédés des sciences de la nature, recommande de substituer «à la multiplicité indéfinie des individus un nombre restreint de types »

1 On peut certes contester, comme l'on fait Durkheim et Mauss (1901), que la propension à classer corresponde à une aptitude naturelle de l'être humain, indépendante de toute insertion sociale. Mais on a pu faire observer que les classifications de la connaissance ordinaire trouvent leur cohérence dans la langue (Degenne, 2005, p. 713). Il n'est donc pas absurde de lier la logique classificatoire à l'apparition du langage articulé, c'est-à-dire à l'aube de l'humanité. 
(1895, 1992, p. 79). Cette idée de réduction de complexité est commune à toutes les disciplines à vocation scientifique. Claude Lévi-Strauss a suggéré à ce propos que les sciences humaines pouvant aspirer au statut de science autrement que par un abus de langage sont celles «qui sont capables de saisir des éléments peu nombreux et récurrents, diversement combinés dans un grand nombre de systèmes, derrière la particularité temporelle et locale de chacun» (1973, p. 345). La réduction de complexité se concrétise par la sélectivité dans les éléments pris en considération. Chaque discipline a ses variables pertinentes, qu'il s'agisse des variables dépendantes - celles que, traditionnellement, elle se propose d'expliquer - ou des variables indépendantes - celles qui lui offrent ses modalités d'explication. C'est autour de ces variables que s'organise un principe de pertinence disciplinaire. La sélection ainsi opérée est alors constitutive de l'objet. S'interroger sur les variables pertinentes d'une discipline revient à se demander comment cette discipline construit son objet. Plus il y a accord sur le choix des variables, plus il y a consensus sur la définition de l'objet. Depuis plusieurs décennies, on a le sentiment que certaines sciences humaines - la science économique, la démographie, la linguistique, l'ethnologie même - ont accepté plus facilement que d'autres l'idée de sélection de quelques dimensions privilégiées aboutissant à un objet relativement facile à isoler. La sociologie, discipline fourre-tout par excellence, ne peut se prévaloir d'un consensus solide sur la définition de son objet. Elle ne suscite l'accord ni sur la classification des variables dépendantes ni sur l'énumération des variables ayant une vocation particulière à être des variables indépendantes. Tout au plus peut-on déceler dans le choix des variables indépendantes une préférence pour les variables structurelles, c'est-à-dire celles qui expriment l'appartenance d'une individu à une catégorie, celles qui situent un individu au sein d'une structure ou encore celles qui expriment les modalités des structures elles-mêmes.

Quelles que soient la rigueur et la précision dans la définition de l'objet, dans les sciences de la nature comme dans les sciences humaines, la sélectivité fondée sur des variables tenues pour pertinentes définit un mode d'explication entre les extrêmes de la contingence pure et du déterminisme absolu. Ce serait une erreur d'associer purement et simplement le déterminisme aux phénomènes naturels et la contingence aux phénomènes sociaux. Pour la biologie moderne, la biosphère est «imprévisible» au même titre qu'une «configuration particulière d'atomes » constituant un caillou ramassé au hasard et l'apparition de la vie sur la terre est un phénomène aléatoire, ce qui suggère à Jacques Monod que «toutes les religions, presque toutes les philosophies, une partie même de la science, témoignent de l'inlassable, héroïque effort de l'humanité niant désespérément sa propre contingence » (Monod, 1970, p. 55). A l'inverse, la part de contingence présente dans les phénomènes sociaux - et qui donne lieu à l'histoire humaine - est limitée par des contraintes structurelles agissant comme des formes transhistoriques et transculturelles s'imposant aux processus qui font l'histoire. On peut donc être tenté de rechercher l'unité des sciences dans un probabilisme mettant en jeu des combinaisons différentes de contingence et de déterminisme. Cependant, le hasard qui intervient dans les phénomènes naturels diffère le plus souvent du hasard qui intervient dans les phénomènes sociaux. Dans le premier cas, les incertitudes les plus fréquentes sont d'ordre opérationnel. Elles résultent de l'impuissance à reconstituer ou à prévoir le déroulement exact des processus dans toute leur 
précision; théoriquement, elles pourraient être éliminées grâce à des instruments plus performants. Dans le second cas au contraire, les incertitudes qui affectent le cours des actions humaines ont un caractère essentiel. Ces incertitudes sont dues à l'intersection de chaînes causales indépendantes (Monod, ibid., p. 128). Il s'agit au surplus de chaînes causales accordant le statut de causes aux raisons des acteurs, c'est-à-dire à des manifestations de volonté humaine.

Les sciences humaines se séparent donc des sciences de la nature face au hasard et c'est ici que s'efface le sentiment d'unité de la démarche scientifique. Les sociologues - et ils ne sont pas rares - qui s'obstinent à vouloir copier le modèle des sciences de la nature ne font que nier la conscience et la liberté humaines. Les sciences de la nature progressent vers des propositions à valeur universelle en traitant les incertitudes opérationnelles comme autant de limites naturelles à la connaissance humaine à une époque donnée. Les sciences humaines partagent l'aspiration à des vérités universelles mais ne s'affranchissent que très malaisément - et toujours partiellement - des contextes historiques ou des contextes locaux car elles sont aux prises avec un autre type d'incertitudes. La contingence y est le résultat de l'autonomie des sujets.

\section{LE SUJET ET L'OBJET}

Les différences entre les deux groupes de disciplines se manifestent dans les modes de conceptualisation et dans le rôle des valeurs dans l'analyse. Conceptualisation et valorisations qui sont étroitement liées peuvent toutefois être examinées sous l'angle de la relation du sujet connaissant à l'objet de sa connaissance. Dans une optique piagétienne, c'est la connaissance elle-même - vraie ou fausse - qui résulte d'une relation entre trois éléments: un sujet, un objet et une structure opératoire (Piaget, 1967, pp. 3-4). Cette structure peut être constituée de classes et d'emboîtements de classes, ou encore de relations, fonctionnelles ou autres. Une structure de ce genre - une structure de connaissance - est présente dans toute relation cognitive établie entre un sujet et un objet. Dans le domaine des sciences humaines, le caractère problématique de la relation entre le sujet et l'objet peut être localisé dans cette structure qui reste imprégnée de subjectivité en dépit de tous les efforts d'objectivation. L'élément de subjectivité se manifeste de plusieurs façons. Tout objet social est constitué d'un enchevêtrement de relations interindividuelles dont les termes - les individus - s'influencent l'un l'autre cognitivement et affectivement. Le chercheur en sciences humaines est lui-même engagé dans un certain nombre de ces relations qui structurent ses perceptions à son insu et qui véhiculent des valorisations. Lorsqu'il applique ses capacités cognitives à un objet social en visant au «degré maximum d'objectivité » possible (Goldmann, 1967, p. 995), il ne peut pas ne pas être le jouet de perceptions sélectives: c'est notamment ce que j'ai appelé «l'impression dominante» dans le contact entre l'observateur participant et son terrain (Coenen-Huther, 1995, chap. 2). Mais l'objet social quel qu'il soit (groupe, réseau, organisation, institution, etc) est fait d'un ensemble d'individus doués de conscience. La démarche du chercheur décrivant, analysant, commentant, publiant ses résultats, constitue une action sur l'objet étudié qu'il ne laisse pas inchangé, à l'encontre de 
l'objet matériel des sciences de la nature. Au surplus, l'objet social peut exercer une action en retour sur le chercheur qui a choisi de l'étudier. En sciences humaines, la relation entre le sujet et l'objet de la connaissance est donc constituée d'une dialectique subtile entre des individus capables de s'influencer les uns les autres, même si les possibilités d'influence ne sont pas symétriques. Le jeu de ces actions et réactions en sens divers est un obstacle à la constitution d'une science du social fonctionnant sur le modèle des sciences de la nature.

Il est vrai que dans les sciences de la nature comme dans les sciences humaines, le choix de l'objet à étudier s'opère sur un arrière-plan de valeurs. Dans l'un et l'autre cas, la valeur dominante - celle qui oriente les opérations de recherche - est la valeur de vérité, pouvant seule accréditer une intention de science. Mais dans les controverses qui agitent les sciences humaines et qui pèsent sur leurs applications, la valeur de vérité se heurte à d'autres valeurs qui lui sont irréductibles, à savoir des valeurs morales porteuses d'antinomies internes: le bien, le juste. Les diverses représentations de la «bonne société» ou des rapports sociaux «équitables» affaiblissent la force normative de la valeur du vrai dans l'étude de la vie en société. De telles représentations - issues de la dévotion à des valeurs concurrentes - constituent au sein même d'un champ disciplinaire, des facteurs internes de subversion du projet scientifique. Au contraire, les controverses qui affectent les sciences de la nature n'ébranlent pas de l'intérieur la dévotion à la valeur de vérité et la conviction de l'unicité de celle-ci. Qu'il s'agisse du débat sur la conception galiléenne du mouvement des astres ou de la controverse sur les dangers des organismes génétiquement modifiés, c'est le vrai - ou le nonvrai - qui reste en cause. Le vrai peut être provisoirement contesté au nom de préoccupations extérieures à la recherche scientifique mais les conventions portant sur les procédures de vérification de la connaissance n'en sont pas durablement affectées. Ce sont ces procédures qui, en fin de compte, tranchent les débats. Une fois que l'évidence s'impose, il y a rétablissement d'un consensus autour de l'unicité du vrai. Car le vrai, à la différence des autres valeurs ultimes, ne comporte aucune antinomie interne susceptible de diviser les esprits et ne dépend d'aucune contingence liée à l'action (Dupréel, 1939, pp. 240-245).

\section{LES INTERROGATIONS FONDAMENTALES}

Les différences qui viennent d'être évoquées ne tiennent pas à un manque de maturité des sciences humaines mais bien à la nature des questions posées. Les sciences de la nature ont affaire à des objets dont la stabilité est incomparablement plus grande que celle des objets sociaux. Il s'agit également d'objets qui ne réagissent pas à l'investigation scientifique comme peuvent le faire les objets sociaux. Il en résulte une stabilité dans le questionnement et l'orientation vers la recherche de lois générales. Il en résulte également une relative indifférence aux réactions de l'environnement social. Les praticiens des sciences de la nature peuvent présupposer sans trop de problèmes que la recherche de lois du fonctionnement de l'univers est une fin en soi (Ringer, 2000, p. 138). Il est vrai que cette présupposition se heurte à certaines réticences depuis l'effondrement des illusions scientistes du $\mathrm{XIX}^{\mathrm{e}}$ siècle mais ces réticences peuvent être disqualifiées comme des réactions 
irrationnelles grâce à la très grande efficacité des sciences en question. Les sciences de la nature se situent donc dans une perspective d'accumulation et d'expansion même si des révolutions paradigmatiques suscitent de loin en loin un sentiment de rupture. La rupture - quand rupture il y a - introduit un progrès dans les connaissances. Les questions posées à l'ordre naturel trouvent des réponses de plus en plus adéquates dont témoignent leurs applications pratiques. Il en va tout autrement des sciences humaines. Elles se trouvent confrontées à des objets qui se transforment à l'échelle d'une vie. Leurs investigations contribuent d'ailleurs dans une certaine mesure à les transformer sans que ces transformations ne soient nécessairement prévues ni souhaitées : il s'agit souvent d'effets pervers. Parmi ces effets pervers les plus notables, il faut mentionner - s'agissant de la sociologie la tendance à l'exonération des responsabilités individuelles par la mise en évidence des causes structurelles des comportements et la généralisation d'un relativisme culturel sapant le respect des valeurs (Simon, 2001, p. 66). L'un et l'autre, outre qu'ils ont un effet paralysant sur certaines prises de décision, remettent en question les diagnostics sociologiques eux-mêmes dans la mesure où ceux-ci sont considérés comme des opinions parmi d'autres. Ainsi, l'objet social transformé induit de nouvelles questions sans que les précédentes n'aient trouvé une réponse indiscutable. Loin de s'insérer dans une perspective d'accumulation, les sciences humaines, et tout particulièrement la sociologie qui en constitue l'exemple extrême, obéissent à des mouvements cycliques au cours desquels des questions émergeant d'une tradition philosophique ancienne sont remises à l'ordre du jour alors que des problèmes très actuels ne font que rarement l'objet de diagnostics convaincants pour tout un chacun. Il n'y a pas de consensus sur les questions à poser à la vie en société, soit que ces questions paraissent superflues, soit qu'elles paraissent incongrues. Car les sciences humaines manifestent une grande sensibilité aux influences de l'environnement social et sont fortement sujettes à des effets de modes. Cette situation particulière des sciences humaines vis-à-vis de leur objet et de leur environnement entraîne des conséquences pouvant être analysées du point de vue de la conceptualisation et du point de vue des valorisations incorporées implicitement ou explicitement dans cette conceptualisation.

\section{LA CONCEPTUALISATION EN SCIENCES HUMAINES}

Lorsqu'on examine plus attentivement l'idée de réduction de complexité évoquée plus haut, on se rend compte qu'elle suscite des procédés de classification différents dans les sciences de la nature et dans les sciences humaines, en particulier en sociologie. Dans les sciences de la nature, quels que soient les critères adoptés, il n'y a pas de raison de ne pas s'en tenir au principe aristotélicien de classement par le genre prochain et la différence spécifique. En sociologie, la réduction de complexité s'appuie sur des éléments d'appréciation extérieurs au principe même du classement ${ }^{2}$. Ceci apparaît à l'examen de traditions sociologiques différentes.

2 J'ai développé ce qui suit dans mon article Compréhension sociologique et démarches typologiques sur lequel je m’appuie fortement ici (Coenen-Huther, 2006). 
Pour Durkheim, il y a lieu de procéder par définition de «types sociaux». Selon lui, le type social doit être fondé sur des faits «cruciaux» ou «décisifs» qui lui confèrent un aspect privilégié dans l'analyse. Ce qui définit à ses yeux ce caractère «crucial» ou «décisif», c'est son aptitude à s'insérer dans une classification des «espèces sociales » s'inspirant des principes taxinomiques des sciences naturelles. On se trouve face à la mise en œuvre d'une morphologie sociale clairement inspirée de la classification des espèces naturelles. Le procédé semblait permettre le recours à un principe de sélectivité interne au corpus de théorie sociologique en voie d'élaboration. Durkheim est néanmoins très attaché à opérer une distinction entre le normal et le pathologique. La typologie, dans son œuvre, a clairement pour objet d'introduire dans l'étude des faits sociaux une distinction entre le normal et l'anormal en évitant de conférer à ces termes une dimension normative. Il s'impose donc de faire usage de cette distinction tout en la relativisant. Dès lors, le type social constitue le niveau intermédiaire entre l'humanité prise globalement et chaque société en particulier. Il permet de relativiser la normalité tout en lui attribuant une signification pouvant être qualifiée de statistique; c'est la fréquence d'apparition d'un phénomène dans une société donnée qui définit son caractère normal ou anormal. Le normal est ainsi défini en dehors de toute valorisation consciente mais il introduit subrepticement un élément de valorisation extra-scientifique. Les faits cruciaux - ces instruments de réduction de complexité - servent à caractériser un type de sociétés mais servent aussi à distinguer le normal du pathologique ou jugé tel. La pensée morale rejoint ici le projet scientifique et l'on s'écarte qu'on le veuille ou non de la pensée nomologique caractéristique des sciences de la nature (Coenen-Huther, 1997a, pp. 121 et ss).

Si Durkheim, tout en s'inspirant de la classification des espèces naturelles, introduit un élément d'ambiguïté dans la typologie des espèces sociales, Max Weber s'écarte plus encore du principe de classement aristotélicien par l'accentuation délibérée du type idéal. Ce mode de conceptualisation correspond à une volonté de stylisation de la réalité, étrangère aux procédés de conceptualisation des sciences de la nature. En l'adoptant à la suite de Montesquieu et de Tocqueville, Weber opte pour ce qu'on appelle de nos jours une logique de modélisation (Coenen-Huther, 2003, pp. 540 sqq.). Le type idéal, comme le modèle, ne décrit pas la réalité empirique mais reflète un construit mental. Celui-ci n'entretient une relation avec le monde réel que moyennant un certain nombre de conditions qui doivent être spécifiées dans chaque cas. La congruence avec des faits empiriquement observables est du domaine du possible mais ce sont les écarts constatés entre la réalité et le type idéal qui ont les vertus heuristiques décisives. L'accentuation unilatérale produite par la conceptualisation idéal-typique résulte de la priorité accordée à l'un ou l'autre aspect de l'objet considéré. Cet élément de sélectivité consciente dépend d'une décision du chercheur et confère inévitablement un caractère historiquement daté à l'analyse. C'est la nature de l'objet - faits naturels ou événements historiques - qui est à la base du contraste entre les catégories de classification immuables des sciences de la nature et la démarche typologique du sociologue. 


\section{CLASSIFICATION ET TYPOLOGIE}

La différence entre les procédés de classement des sciences de la nature et ceux des sciences humaines a été mise en évidence par Jean-Claude Passeron. Celui-ci en conclut qu'il est préférable d'inscrire cette différence dans la terminologie en réservant le terme de typologie aux classifications des sciences humaines. Il est tout à fait possible de se rallier à cette convention de langage en se rappelant néanmoins qu'elle est loin d'être acceptée par tous les sociologues. Alain Degenne, dans le Dictionnaire de la pensée sociologique, note que «l'usage consacre indifféremment typologie, classification, taxonomie, voire nomenclature, même si certains auteurs cherchent à réserver chaque terme à une méthode particulière (2005, p. 713). Demazière et Dubar utilisent le terme de typologie comme synonyme de classification (1997, p. 274). Pour Boudon et Bourricaud, dans le Dictionnaire critique de la sociologie, la seule chose qui distingue une typologie d'une classification est le nombre de critères utilisés pour distinguer les éléments d'un ensemble. Lorsque la répartition se base sur un critère unique, on parle de classification; lorsqu'on utilise plusieurs critères, il peut être question de typologie. Il en résulte, pour ces auteurs, que « les typologies ne sont qu'une forme particulière de classification» (1982, p. 578).

Mais en cela comme en d'autres matières, les concepts comptent beaucoup plus que les mots qui servent à les désigner. Jean-Claude Passeron voit dans la classification et la typologie le résultat de deux méthodes distinctes. Pour lui, la classification résulte d'une démarche inductive inspirée du modèle des sciences de la nature alors que la typologie est le fruit d'une méthodologie idéal-typique liée «à la forme de présentation des phénomènes dans l'observation historique » (Passeron, 1994, pp. 7-8). On se trouve ainsi face à deux conceptions opposées des procédés classificatoires. La première, d'orientation positiviste, s'en remet à une «classification générique » à laquelle seule une «représentation nomologique des généralités historiques » peut conférer un potentiel opératoire. La seconde, inspirée par une théorie de la connaissance d'inspiration néo-kantienne, développe une méthodologie idéal-typique en réaction aux illusions d'une «épistémologie mimétique des sciences de la nature» (ibid., pp. 6-9). Pour Passeron, lorsque l'analyse sociologique se fonde sur l'observation historique, elle présente ses énoncés «dans un langage dont les assertions ne doivent leur généralité conceptuelle qu' à leur forme typologique» (ibid., p. 5; souligné par JCH). Cette prise de position repose sur une conception de la sociologie comme discipline hybride, oscillant entre un pôle historique et un pôle expérimental $(1991,2006)$. Une discipline est historique, estime Passeron, lorsque les propositions qu'elle énonce ne peuvent être décontextualisées; en d'autres termes, lorsqu'elles ne peuvent être séparées «des contextes dans lesquels sont prélevées les données ayant du sens pour elle» (1990, p. 15). Le raisonnement sociologique présente souvent ce caractère historique mais il se distingue du raisonnement purement historique par « des moments de raisonnement expérimental» alternant avec des phases de « raisonnement naturel». C'est un raisonnement «mixte» qui ne peut que se déplacer entre les deux pôles dans la mesure où il se fonde sur «des faits datés et localisés » mais s'efforce d'échapper à «cette datation et cette localisation» en raison de sa visée généralisante (ibid. pp. 15-16). La volonté de généralisation du sociologue ne peut 
néanmoins s'appuyer que sur des procédures fragiles au regard de la méthode expérimentale. Et si la stratégie comparative propre à la sociologie peut malgré tout prendre une dimension transculturelle ou transhistorique c'est grâce à la construction de types idéaux. Que l'on songe, par exemple, à l'opposition aristocratie-démocratie dans l'œuvre de Tocqueville. Lorsque celui-ci cherche une approximation historique du type aristocratique pur, il se tourne vers la France du $\mathrm{XI}^{\mathrm{e}}$ siècle. Cherchant alors la meilleure approximation du type démocratique, il la trouve dans les Etats-Unis de son temps ${ }^{3}$. Seule l'adhésion à un mode de conceptualisation idéal-typique rend acceptable une stratégie comparative basée sur un tel voyage dans le temps et dans l'espace.

Cette conception des procédés de conceptualisation caractéristiques des sciences humaines pose le double problème de la compréhension et de la distanciation. Il est généralement admis de nos jours qu'un processus d'interprétation fondé sur la compréhension d'actions individuelles constitue un moment obligé de toute recherche en sciences humaines. La mise en relation de grandeurs agrégées ne trouve son sens que par l'enchaînement de séquences comportementales plausibles. Et la plausibilité ne prend un caractère d'évidence que lorsque la logique de l'acteur devient accessible à l'observateur. Cette opération mentale d'élaboration de sens qu'on appelle «compréhension» présuppose qu'il soit possible au chercheur de surmonter la distance sociale ou culturelle, voire même la distance historique qui le sépare éventuellement de l'acteur. Mais c'est ce pari sur l'existence d'invariants du comportement humain, permettant de comprendre les mobiles de l'acteur par identification à autrui et projection de raisons d'agir, qui fait de l'inévitable compréhension un maillon faible de l'analyse sociologique ${ }^{4}$. En effet, la compréhension repose au moins en partie sur l'application d'une généralisation basée sur notre propre expérience ou sur notre sens personnel de l'évidence. Le risque est grand d'attribuer à l'acteur une logique de comportement dont on juge la portée universelle alors qu'elle est liée à un contexte socio-culturel particulier. Seul un optimum d'identité et d'altérité permet d'éviter la projection abusive du point de vue de l'observateur tout comme l'adhésion non critique à celui de l'acteur. Mais il n'est guère de recette standardisée permettant d'atteindre cet optimum ni même de le définir. Raymond Aron fait observer à ce sujet que «le sociologue ne peut comprendre une société sans user d'un schéma conceptuel. Or s'il utilise son propre schéma conceptuel, il comprendra la société étrangère autrement que celle-ci ne s'est comprise elle-même et du même coup, il en faussera la signification» (1959, p. 34). Pour Lucien Goldmann, la distance cognitive et affective entre l'observateur et son objet doit être appréciée dans chaque cas particulier. Il évoque à cet égard les cas de figure respectifs d'un sociologue français étudiant la vie des esquimaux ou les transformations de la classe ouvrière française. Il faut, écrit Goldmann, « dégager autant que possible le degré spécifique d'identité entre le sujet et l'objet et par cela même le degré d'objecti-

3 J'ai présenté ceci plus en détail dans mon Tocqueville (1997b, chap. III, « Tocqueville, comparatiste avisé », pp. 52-78).

4 Voir à ce sujet mon article Formes et ambiguités de la compréhension en situation d'observation dans lequel je m'appuie sur des expériences de terrain (Coenen-Huther, 1998, pp. 77-96) 
vité accessible à la recherche» (1967, pp. 995-996). Cet auteur nous renvoie ainsi à une difficulté réelle d'objectivation de la relation sujet-objet. La structure de connaissance est ouverte à la subjectivité. Et si la distance par rapport à l'objet est une variable, il en va de même de la capacité à adopter une attitude de neutralité axiologique par rapport à cet objet.

\section{LE RÔLE DES VALEURS}

La méthodologie idéal-typique implique une décision relative au point de vue adopté sur l'objet social étudié. Ainsi, l'objet «capitalisme» peut être caractérisé par la maximisation du profit, par la rationalisation des procédés ou par la propension à innover. Le choix du critère dépend de la capacité de compréhension de comportements jugés typiques. Compte tenu de la perméabilité des sciences humaines aux influences de leur environnement social, rien ne garantit que ce choix soit exempt de valorisations positives ou négatives. Dès lors, il est difficile de s'en tenir à l'idée d'une séparation stricte entre jugements de fait et jugements de valeurs. L'attitude à adopter à l'égard de ce postulat positiviste a fait l'objet de nombreuses controverses parmi les sociologues. Entre les extrêmes de l'acceptation ou du rejet de ce postulat, on trouve des points de vue plus nuancés, visant à préserver la possibilité d'une démarche authentiquement scientifique face à l'intrusion de valeurs autres que la valeur de vérité. Une distinction est ainsi proposée entre le choix d'un objet d'étude et la constitution de cet objet. Pour Georges Goriely, «si les valeurs sont constitutives d'un point de vue, et d'une sélection, elles ne sont pas constitutives de l'objet même de l'investigation» (1950, p. 31). Il faut néanmoins faire observer à ce propos que le point de vue adopté est bel et bien constitutif de l'objet dans la mesure où il guide les questions qui seront posées à la réalité étudiée et qui orientent la connaissance; le capitalisme de Karl Marx n'est ni celui de Max Weber ni celui de Schumpeter.

La tentative la plus systématique de faire la part du feu et de s'accommoder de l'irruption des valeurs dans le champ scientifique est le raisonnement weberien fondé sur la distinction entre jugement de valeur (Werturteil) et rapport aux valeurs (Wertbeziehung). Pour Max Weber, la sélection par les valeurs est un fait acquis. Les valeurs échappent au discours rationnel car elles sont l'expression de nos sentiments ou de notre volonté. La sélection - qui est constitution d'objet - dépend donc d'une volonté libre: celle de l'historien ou du sociologue. S'agissant des sociologues ou des historiens, Raymond Aron souligne que «les concepts qu'ils emploient, et par suite les ensembles qu'ils organisent, dérivent des questions qu'ils posent à la matière» $(1938,1950$, p. 255). La sélection au départ de toute investigation a donc un caractère subjectif mais il n'en reste pas moins un espace pour la recherche de la vérité avant et après cette sélection (Aron, ibid., p. 221).

Max Weber s'applique à montrer comment il est possible de se laisser inspirer par des valeurs dans l'étude des faits sociaux tout en s'abstenant d'introduire des jugements de valeurs dans l'analyse. Soit l'exemple de la Révolution française. Voilà bien un objet marqué par l'affrontement de prises de position 
contradictoires ${ }^{5}$. On peut y voir un progrès décisif vers la liberté humaine mais on peut y voir également l'émergence d'une nouvelle forme de despotisme. On peut donc faire l'histoire des événements révolutionnaires en prenant pour point de départ et pour repère la valeur «liberté ». Dès lors, les faits seront sélectionnés et soupesés en fonction d'un diagnostic à poser sur la signification historique de la Révolution française pour la réalisation de la liberté. Dans ce cas, la valeur «liberté» est donc bien «l'un des concepts à l'aide desquels l'historien choisit, construit, organise la réalité à étudier » (Mesure et Renaut, 1996, p. 102). Ce choix n'est pas indépendant du contexte socio-historique. L'examen du rapport entre la Révolution française et la liberté correspond aux préoccupations de l'historien en tant qu'homme de son temps et de son milieu; il correspond également aux questions qu'on se pose autour de lui à son époque. Une échelle de valeurs commande donc la constitution de l'objet d'étude. Les prémisses ainsi posées, l'analyse peut néanmoins offrir toutes les garanties d'honnêteté intellectuelle et être exempte de jugement de valeur bien qu'un «rapport aux valeurs» soit manifeste. Pour Gonthier, le rapport aux valeurs représente « un moyen terme cognitif entre ce qui n'a pas de signification (sinnlos) et ce qui est significatif (sinnhaft), entre ce qui n'a pas de sens et ce qui est essentiel » (2006, p. 19). Il serait bon de préciser que ce qui est significatif ou dénué de signification ne peut l'être que par rapport à un projet intellectuel donné.

\section{FRAGILITÉ DE LA THÈSE WEBERIENNE}

La démarche weberienne en la matière est cependant fragile. La distinction entre jugements de valeurs et jugements de fait est souvent beaucoup moins nette qu'une vulgate sociologique tend à le supposer. L'objet «totalitarisme» est défini en creux, à partir d'une conception valorisée de l'Etat de droit de type libéral. Sur la base de critères formels, il est alors possible de mettre sur le même plan le régime stalinien et le régime nazi. L'existence d'un système concentrationnaire de part et d'autre tend à renforcer l'idée de similitude entre les deux régimes. Mais il est également possible de faire intervenir des éléments relatifs au projet sociopolitique de l'un et l'autre régime. Sur cette base, des différences peuvent être mises en évidence. Le choix des arguments en la matière n'est pas axiologiquement neutre; il est généralement cohérent avec des appréciations plus générales portant sur les différents régimes politiques. A l'affirmation «un camp est un camp» s'oppose le jugement selon lequel la coercition au service d'un projet de réaménagement social n'est pas assimilable à la coercition au service d'un projet de domination raciale. La sélectivité dans le choix des critères n'est plus ici un choix méthodologique, c'est le résultat d'une perception sélective influencée par des jugements de valeurs.

Max Weber lui-même a fourni des arguments à l'appui de la précarité de sa thèse. Il va de soi, estime-t-il, qu'on ne peut guère faire de l'histoire ou de la socio-

5 Cet exemple est décrit et commenté par Sylvie Mesure et Alain Renaut dont je suis ici de près l'argumentation (Mesure et Renaut, 1996, p. 102). 
logie de l'art si l'on ne possède pas «la faculté de comprendre la production artistique». Et il est tout aussi évident que cette faculté est inconcevable «sans la faculté d'apprécier» (1965, p. 453). Ce raisonnement weberien peut s'appliquer à d'autres domaines. Pour l'historien, note Raymond Aron, le sens attribué aux actes du régime de Vichy dépend du jugement porté sur «l'option prise par le maréchal Pétain en $1940 »$; ce jugement est incontestablement un jugement de valeur: on jugera cette option «courageuse et lucide» ou au contraire «lâche et irréfléchie» (Mesure et Renaut, ibid., p. 104). La critique la plus vigoureuse de la position weberienne en matière de valeurs est celle de Leo Strauss. Attaché à la notion de droit naturel - et peu réceptif à la spécificité de la démarche sociologique -, celui-ci voit dans les conceptions de Weber une forme de relativisme conduisant logiquement au nihilisme $(1954, \text { p. 58) })^{6}$. En particulier, le «rapport aux valeurs» lui paraît incompatible avec la neutralité axiologique. Leo Strauss prend pour point de départ de son raisonnement critique l'idée qu'une connaissance scientifique du social (ou du politique) présuppose toujours une connaissance pré-scientifique. La connaissance scientifique est donc une connaissance «seconde ou dérivée » par rapport à la connaissance ordinaire. Mais le sens commun ne fait pas de distinction nette entre les faits et les valeurs: «la distinction entre faits et valeurs est étrangère à la compréhension des choses politiques propre au citoyen» (2001, p. 99). Certes, la compréhension «spécifiquement scientifique i implique «une rupture avec la compréhension pré-scientifique » (ibid.) et c'est alors que se manifeste la volonté de bien marquer la limite entre faits et valeurs. Il n'en reste pas moins que la connaissance scientifique du social, en raison de ses racines dans la connaissance ordinaire, est en quelque sorte contaminée par la confusion entre faits et valeurs entretenue par le sens commun. Pour étayer sa démonstration, Leo Strauss tente de montrer que Max Weber lui-même n'a pas pu respecter ses propres principes de méthode dans sa pratique de sociologue. «L'œuvre de Weber», écrit-il, «aurait été non seulement ennuyeuse mais absurde s'il n'eût parlé à tout bout de champ des vertus et des vices intellectuels et moraux dans le registre approprié, celui de la louange ou du blâme» (1954, p. 67). Le fait même de parler de «vide spirituel» pour caractériser une époque implique un jugement de valeur. De même, lorsque Weber met en relation «une religion authentique et supérieure» avec «la décadence de l'art», il fait certes œuvre de sociologue mais il fonde son raisonnement sur deux jugements de valeur (1954, p. 68) et ceci n'est pas seulement une question de vocabulaire.

En fait, pour Leo Strauss, une science sociale ou politique qui se voudrait entièrement exempte de jugements de valeurs se condamnerait à la futilité. En effet, écrit-il, «que deviendrait la science politique s'il n'était pas permis de s'intéresser à des phénomènes comme l'esprit de clique, le patronage électoral, les coalitions d'intérêt, l'art de l'homme d'Etat, la corruption et même la corruption morale, autant de phénomènes qui sont faits, somme toute, de jugements de valeur?». Une pseudo-solution rencontrée fréquemment consiste à «mettre entre

6 Voir à ce sujet les commentaires de Pierre-Jean Simon (2001, p. 66) et de Nicolas Tenzer (1994, pp. 627-628) ainsi que la réfutation de Nathalie Heinich qui rejette un peu rapidement l'argumentation de Strauss comme «sophistique» (2006, p. 293). 
guillemets les termes qui désignent ces réalités », de façon à suggérer une prise de distance. Mais notre auteur n'y voit qu' «un truc enfantin» (1954, p. 79). Ce qui est en jeu est la possibilité pour le chercheur de présenter des conclusions qui aient quelque pertinence pour ses contemporains.

\section{VALEURS ET ARGUMENTATION}

De quelle légitimité peuvent bénéficier les sciences humaines si elles ne peuvent opérer une distinction convaincante entre faits et valeurs? La notion de «connaissance dérivée» proposée par Leo Strauss peut nous être d'un certain secours pour répondre à cette question. Les raisonnements proposés par les sciences humaines se situent dans le prolongement de la connaissance ordinaire, soit pour la préciser et la nuancer, soit pour la remettre en question et souligner ses aspects fallacieux. Ces disciplines jouent donc un rôle de remise en question des évidences du sens commun. Les sciences humaines sont en discussion permanente avec la connaissance ordinaire qui les inspire ou les stimule. Elles argumentent autant qu'elles interprètent ou expliquent. Illustrons ceci par une anecdote typique des régions de haute montagne. Un homme très âgé habite un chalet isolé, situé sur une hauteur escarpée. Les pentes qui conduisent à la route la plus proche sont d'accès malaisé à la descente comme à la montée. La famille de notre homme, soucieuse de lui faciliter ses déplacements, envisage de lui aménager un sentier plus praticable, de façon à préserver son autonomie le plus longtemps possible. Mais le sentier en question devrait traverser des terrains appartenant à une autre famille de la région. Les propriétaires refusent leur accord, même à titre temporaire, alors que la parcelle visée ne fait l'objet d'aucune exploitation, ni agricole ni forestière. Voilà un épisode de la comédie humaine qui est bien de nature à alimenter des rancunes tenaces sur le plan local. Présenté sans autre commentaire, il ne peut qu'être considéré comme une manifestation d'égoïsme gratuit et susciter la réprobation. Intervient alors un sociologue ou un anthropologue. Celui-ci fait observer que le refus est provoqué par la crainte de créer à terme une servitude de passage qui ne soit plus liée à des circonstances particulières, c'est-à-dire un précédent faisant en quelque sorte jurisprudence en droit coutumier. Par son diagnostic, le praticien des sciences sociales opère la mise en perspective d'une décision jugée critiquable et absout les propriétaires du terrain de l'accusation de mauvaise volonté irraisonnée. Nous nous trouvons ici devant une démarche argumentative. Il y a, symboliquement parlant, dialogue avec le sens commun. L'argumentation neutralise au moins partiellement des jugements moralisateurs et suggère implicitement que - dans un contexte d'affaiblissement relatif du droit de propriété - il existe un risque réel pour les propriétaires. Le raisonnement implique une prise de distance à l'égard des réactions spontanées du sens commun mais il n'en accepte pas moins la notion de propriété privée. Ce raisonnement n'est recevable que dans un contexte où la propriété privée est une valeur. La réaction d'indignation initiale peut être calmée pour autant que les destinataires de l'argumentation valorisent la propriété privée et partagent le sentiment que toute servitude de passage est un danger contre lequel il importe de se prémunir. Si les interlocuteurs ne valorisent pas la propriété privée et sont indifférents au risque qu'elle peut courir, la contextualisation devient inopérante et le raisonnement s'avère dénué de pertinence. $\mathrm{Au}$ 
départ de l'argumentation sociologique, une valeur est posée implicitement - et même inconsciemment - à savoir la valeur de la propriété privée. Si la valorisation de la propriété privée n'est pas rendue explicite, c'est parce qu'elle est tacitement présupposée.

\section{POUR CONCLURE}

Le praticien des sciences humaines prend appui sur des valeurs qui sont les siennes mais qui sont également celles de la population à laquelle il s'adresse mentalement: ses concitoyens, ses contemporains, les gens de son époque. Son ambition d'atteindre un auditoire universel est contrecarrée par les mêmes obstacles que l'opération de compréhension assortie d'une intention généralisante. Elle apparaît illusoire lorsque les circonstances le mettent en présence d'individus qui ne partagent en aucune façon ses valorisations implicites. De façon plus générale, les sciences humaines sont appelées à intervenir dans le débat public. Elles argumentent face à des profanes qui ont un accès direct aux faits et qui ont des opinions plus ou moins fondées sur eux. Le mélange de descriptions et de valorisations caractérisant la connaissance ordinaire rejaillit sur le discours à visée scientifique qui se greffe sur elle, porteur d'une connaissance seconde. L'intrusion fréquente de valeurs dans la conceptualisation des sciences humaines ne se produit pas seulement sous la forme de rapports aux valeurs mais également - ce qui est intellectuellement beaucoup moins confortable - sous la forme de jugements de valeurs incorporés inconsciemment ou implicitement aux raisonnements. $\mathrm{Ce}$ sont ces valorisations qui réduisent dans le temps et dans l'espace la portée de propositions dont l'ambition à l'universalité est toujours renaissante mais toujours compromise.

Département de Sociologie

Université de Genève

\section{RÉFÉRENCES BIBLIOGRAPHIQUES}

Aron, Raymond, La philosophie critique de l' histoire, Paris, Vrin, 1938, $2^{\mathrm{e}}$ édition, 1950.

Aron, Raymond, «Introduction», in: Max Weber, Le savant et le politique, Paris, Plon, Coll. «10/18», 1959.

Boudon, Raymond et Bouricaud, François, Dictionnaire critique de la sociologie, Paris, PUF, 1982. Coenen-Huther, Jacques, Observation participante et théorie sociologique, Paris, L'Harmattan, 1995.

Coenen-Huther, Jacques, «Durkheim et l'impératif de réduction de complexité », in: Ch.-H. Cuin, Ed., Durkheim d' un siècle à l'autre, Paris, PUF, 1997a, pp. 119-130.

Coenen-Huther, Jacques, Tocqueville, Paris, PUF, Coll. «Que sais-je?», 1997b.

Coenen-Huther, Jacques, «Formes et ambiguïtés de la compréhension en situation d'observation», Cahiers Internationaux de Sociologie, Vol. CIV, 1998, pp. 77-96.

Coenen-Huther, Jacques, «Le type idéal comme instrument de la recherche sociologique», Revue française de sociologie, 44-3, juillet-septembre 2003, p. 531-547.

Coenen-Huther, Jacques, «Compréhension sociologique et démarches typologiques», Revue Européenne des Sciences Sociales, Tome XLIV, No 135, 2006, pp. 195-205. 
Degenne, Alain, «Typologie», in: Borlandi, Massimo et al., Eds, Dictionnaire de la pensée sociologique, Paris, PUF, 2005, pp. 713-717.

Demazière, Didier et Dubar, Claude, Analyser les entretiens biographiques. L'exemple des récits d'insertion, Paris, Nathan, 1997.

Dupréel, Eugène, Esquisse d' une philosophie des valeurs, Paris, Alcan, 1939.

Durkheim, Emile, Les règles de la méthode sociologique, Paris, 1895, PUF, Quadrige, 1992.

Durkheim, Emile et Mauss, Marcel, «De quelques formes primitives de classification. Contribution à l'étude des représentations collectives », L'Année sociologique, Vol. 6, 1901-1902.

Goldmann, Lucien, «Epistémologie de la sociologie», in: Jean Piaget, Logique et connaissance scientifique, Paris, Gallimard, La Pléiade, 1967, pp. 992-1018.

Gonthier, Frédéric, «Relativisme et vérité scientifique chez Max Weber», L'Année sociologique, Vol. 56, No 1, 2006, pp. 15-39.

Goriely, Georges, «De la relativité du savoir sociologique», Revue de l'Institut de Sociologie, No 1, 1950, pp. 19-34.

Heinich, Nathalie, «La sociologie à l'épreuve des valeurs», Cahiers internationaux de Sociologie, Vol. CXXI, 2006, pp. 287-315.

Lévi-Strauss, Claude, Anthropologie structurale deux, Paris, Plon, 1973.

Mesure, Sylvie et Renaut, Alain, La guerre des dieux. Essai sur la querelle des valeurs, Paris, Grasset, 1996.

Monod, Jacques, Le hasard et la nécessité, Paris, Seuil, 1970.

Passeron, Jean-Claude, «L'enseignement, lieu de rencontre entre historiens et sociologues », débat avec Antoine Prost, Sociétés Contemporaines, No 1, mars 1990, pp. 7-45.

Passeron, Jean-Claude, Le raisonnement sociologique: l'espace non-poppérien du raisonnement naturel, Paris, Nathan, 1991, réédition Paris, Albin Michel, 2006.

Passeron, Jean-Claude, «La rationalité et les types de l'action sociale chez Max Weber», Revue Européenne des Sciences Sociales, Tome XXXII, No 98, 1994, pp. 5-44.

Piaget, Jean, Logique et connaissance scientifique, Paris, Gallimard, Coll. «La Pléiade», 1967.

Ringer, Fritz, Max Weber's Methodology, Cambridge, Mass., Harvard University Press, 1997, réimpression 2000.

Schnapper, Dominique, La compréhension sociologique. Démarche de l'analyse typologique, édition revue et augmentée, Paris, PUF, Quadrige, 2005.

Simon, Pierrre-Jean, Eloge de la sociologie ou la fécondité du néant, Paris, PUF, 2001.

Strauss, Leo, Droit naturel et histoire, Paris, Plon, Coll. «Recherches en sciences humaines », 1954.

Strauss, Leo, Nihilisme et politique, Paris, Payot/Rivages, 2001.

Tenzer, Nicolas, Philosophie politique, Paris, PUF, 1994.

Valade, Bernard, «De la classification et des classificateurs», in: M. Cherkaoui, Ed., Histoire et théorie des sciences sociales. Mélanges en l'honneur de Giovanni Busino, Genève, Droz, 2003, pp. 123-135.

Weber, Max, Essais sur la théorie de la science, trad. Julien Freund, Paris, Plon, 1965. 